\title{
Implementation of a Fourier matched filter in CMB analyses
}

\section{Application to ISW studies}

\author{
C. Hernández-Monteagudo
}

\begin{abstract}
Max Planck Institut für Astrophysik, Karl Schwarzschild Str.1, 85741 Garching bei München, Germany e-mail: chm@mpa-garching.mpg.de
\end{abstract}

Received 30 March 2008 / Accepted 11 July 2008

\begin{abstract}
We implement a matched filter (MF) cross-correlation algorithm in multipole space and compare it to the standard angular cross power spectrum (ACPS) method. Then we apply both methods to a integrated Sachs Wolfe (ISW) - large scale structure (LSS) cross correlation scenario. We study how sky masks influence the multipole range where the cross correlation signal arises and compare it to theoretical predictions. The MF requires the inversion of a multipole covariance matrix that, under partial sky coverage $\left(f_{\text {sky }}<1\right)$, is generally non diagonal and singular. We chose a singular value decomposition (SVD) approach that enables identification of those modes carrying most of the information from those more likely to introduce numerical noise (that are dropped from the analysis). We compared the MF to the ACPS in ISW-LSS Monte Carlo simulations, focusing on the effect that a limited sky coverage has on the cross-correlation results. Within the data model $\boldsymbol{s}=\boldsymbol{t}+\alpha \boldsymbol{m}$ where $\boldsymbol{t}$ is Gaussian noise and $\boldsymbol{m}$ is a known filter, we find that the MF performs better than the ACPS for lower values of $f_{\text {sky }}$ and scale-dependent (non-Poissonian) noise fields. In the context of ISW studies, both methods are comparable, although the MF performs slightly more sensitively under more restrictive masks (lower values of $\left.f_{\text {sky }}\right)$. A preliminary analytical study of the ISW-LSS cross correlation signal-to-noise $(S / N)$ ratio shows that most of it should be found on very large scales $(50 \%$ of the $S / N$ at $l<10,90 \%$ at $l<40-50)$, and this is confirmed by Monte Carlo simulations. The statistical significance of our cross-correlation statistics reaches its maximum when considering $l \in\left[2, l_{\max }\right]$, with $l_{\max } \in[5,40]$ for all values of $f_{\text {sky }}$ observed, despite the smoothing and power aliasing that aggressive masks introduce in Fourier space. This $l$-confinement of the ISW-LSS cross correlation should enable a safe distinction from other secondary effects arising on smaller (higher $l$-s) angular scales.
\end{abstract}

Key words. cosmic microwave background - large-scale structure of Universe

\section{Introduction}

Auto and cross-correlation analyses are crucial in the study of the cosmic microwave background (CMB) anisotropies, because the theory can only predict statistical properties of the intrinsic intensity and polarization anisotropies; hence auto-correlation tests must be conducted in order to compare theory to observations, (see Hu \& Dodelson 2002, for a review). They are also crucial because of the presence of secondary anisotropies and foreground emission that add up to the measurements in the microwave range. These other components must be identified and separated from the intrinsic ones generated at the surface of last scattering, and therefore cross-correlation analyses to other data sets probing the sources of this secondary emission must be carried out. This has been done practically for all CMB experiments, from COBE data (Smoot et al. 1992; Bennett et al. 1996) all the way to WMAP data (Bennett et al. 2003; Spergel et al. 2007). These cross correlation techniques may be either based in real space (like the angular two point correlation function), in Fourier space (like the auto and cross angular power spectrum), or in wavelet space (Cayón et al. 2000; Larson et al. 2005).

In the linear theory that characterizes the intensity and polarization anisotropies of the $\mathrm{CMB}$, predictions are made in the Fourier space of the $2 \mathrm{D}$ sphere, that is, in multipole space. In this space, the statistical covariance matrices between different modes are particularly simple, and so is the comparison of theory to observations. It is in this space where theoretical expectations for other secondary effects present in the CMB are also displayed, and where the constraints on the cosmological parameters are set (e.g., Dunkley et al. 2008; Reichardt et al. 2008). However, there are two practical issues that tend to complicate this theory to data comparison: the presence of cosmic variance on large angular scales (that is, the sample variance due to having only one single sky to look at) and the coupling of different Fourier modes whenever only part of the sky is subject to analysis, as happens in practice for current and future CMB and LSS surveys like ACT (Kosowsky 2003), SPT (Ruhl et al. 2004), DUNE ${ }^{1}$, SNAP $^{2}$ etc. These two effects are of particular relevance in the study of the integrated Sachs-Wolfe (ISW) effect (Crittenden \& Turok 1996): the ISW arises on large angular scales, and since its frequency dependence is identical to that of the intrinsic CMB fluctuations, it must be identified via crosscorrelation tests with large scale structure surveys that are likely to cover only a fraction of the sky.

In this work we generalize the matched filter crosscorrelation method to multipole space in the context of CMB studies. We compare it to the standard angular cross power spectrum in different scenarios, and show that the former is either equivalent or superior to the latter. We also perform this comparison in Monte Carlo simulations of the ISW effect, with similar results. The method is developed in Sect. 2, whereas a first

\footnotetext{
1 http://www . dune-mission.net/

2 http://snap. lbl.gov/
} 
comparison to the angular cross power spectrum is given in Sect. 3. A detailed analysis of the signal to noise ratio of ISW cross-correlation measurements is provided in Sect. 4, where the matched filter method is again compared to the angular cross power spectrum. Finally, in Sect. 5 we discuss our results and conclude.

\section{The matched filter method}

\subsection{A brief description}

Our first goal is to estimate the level of presence of some known signal $\boldsymbol{m}$ in some measured data array $\boldsymbol{s}$, which is therefore decomposed as $\boldsymbol{s}=\boldsymbol{t}+\alpha \boldsymbol{m}$. We assume that $\boldsymbol{t}$ is a Gaussian vector (which will be regarded as noise) whose covariance matrix $\mathbf{C}$ is known. Given the Gaussian assumption, $\mathbf{C}$ completely characterizes $\boldsymbol{t}$. As shown in, e.g., Górski et al. (1996), the minimization of the quantity

$\chi^{2} \equiv \sum_{i, j}(\boldsymbol{s}-\alpha \boldsymbol{m})_{i}\left(\mathbf{C}^{-1}\right)_{i, j}(\boldsymbol{s}-\alpha \boldsymbol{m})_{j}$,

yields the following estimates for $\alpha$ and its formal error:

$\hat{\alpha}=\frac{\boldsymbol{t}^{\mathrm{t}} \mathbf{C}^{-} 1 \boldsymbol{m}}{\boldsymbol{m}^{\mathrm{t}} \mathbf{C}^{-1} \boldsymbol{m}}, \quad \hat{\sigma}_{\alpha}^{2}=\frac{1}{\boldsymbol{m}^{\mathrm{t}} \mathbf{C}^{-1} \boldsymbol{m}}$.

Note that the superscript $t$ denotes transpose. The difficulty usually lies in the inversion of the covariance matrix for long data arrays $\boldsymbol{t}$ and/or for close-to-singular covariance matrices $\mathbf{C}$. The first scenario has already been addressed in Hernández-Monteagudo et al. (2006a), where this technique is applied in separated subsets of data, and then the covariance among different subsets computed separately. Here, we also consider the case where $\mathbf{C}$ is singular or close to singular.

Indeed, the use of the matched filter is very extended in CMB analyses (e.g., Rubiño-Martín et al. 2000; HernándezMonteagudo \& Rubiño-Martín 2004; Hernández-Monteagudo et al. 2004; Hansen et al. 2005), but it has been mostly restricted to real space. In works like that of Hansen et al. (2005) it is also implemented in Fourier (multipole) space, but only after approximating the covariance matrix as diagonal, an assumption that we avoid here.

\subsection{The covariance matrix in multipole space}

We will focus our analyses on real signals defined on 2D spheres. These are usually decomposed on a spherical harmonic basis as:

$\boldsymbol{s}(\hat{\boldsymbol{n}})=\sum_{l=l_{\min }}^{l_{\max }} \sum_{m=-l}^{l} a_{l, m} Y_{l, m}(\hat{\boldsymbol{n}})$,

with $\hat{\boldsymbol{n}}$ denoting a direction on the sky (or a position in the sphere). If $s$ is real, then the multipole coefficients verify $a_{l,-m}=$ $(-1)^{m} a_{l, m}^{*}$, with the symbol "*" denoting complex conjugate. This limits the number of degrees of freedom per $l$ to $2 l+1$. The $m=0$ multipole is by definition real, so the $2 l$ remaining degrees of freedom can be assigned to the real and imaginary parts of the $a_{l, m}$ multipoles with magnetic number ranging from $m=1$ to $m=l$; i.e., for a given multipole $l$, the multipole array $a_{l, m}$ will be decomposed into a $2 l+1$ dimensioned array $\left(u_{0}^{l}, u_{1}^{l}, \ldots, u_{l}^{l}, v_{0}^{l}, \ldots, v_{l}^{l}\right)$, where $u_{0}^{l} \equiv a_{l, 0}, u_{1}^{l}, \ldots, u_{l}^{l}$ contain the real parts of $a_{l, m=1, l}$, and $v_{1}^{l}, \ldots, v_{l}^{l}$ the imaginary ones. Since we simultaneously consider all multipoles $l \in\left[l_{\min }, l_{\max }\right]$, we define the multipole array

$\boldsymbol{a}=\left(\boldsymbol{u}_{0}, \boldsymbol{u}, \boldsymbol{v}\right)$ where

$$
\begin{aligned}
\boldsymbol{u}_{0} & \equiv\left(u_{0}^{l_{\min }}, u_{0}^{l_{\min }+1}, \ldots, u_{0}^{l_{\max }}\right), \\
\boldsymbol{u} & \equiv\left(u_{1}^{l_{\min }}, \ldots, u_{l_{\min }}^{l_{\min }}, \ldots, u_{1}^{l_{\max }}, \ldots, u_{l_{\max }^{l_{\max }}}\right),
\end{aligned}
$$

and

$\boldsymbol{v} \equiv\left(v_{1}^{l_{\min }}, \ldots, v_{l_{\min }}^{l_{\min }}, \ldots, v_{1}^{l_{\max }}, \ldots, v_{l_{\max }}^{l_{\max }}\right)$.

The dimension of $\boldsymbol{u}$ and $\boldsymbol{v}$ is given by $n_{l, 2}=l_{\max }\left(l_{\max }+1\right) / 2+$ $l_{\max }+1-\left(l_{\min }\left(l_{\min }-1\right)+l_{\min }\right)$, whereas the dimension of $\boldsymbol{u}_{0}$ is simply $n_{l, 1}=l_{\max }-l_{\min }+1$, so the total dimension of $\boldsymbol{a}$ reads as $n_{l}=n_{l, 1}+2 \times n_{l, 2}$. If $\boldsymbol{s}(\hat{\boldsymbol{n}})$ is an isotropic, Gaussian-distributed signal over the whole sphere $\left(f_{\text {sky }}=1\right)$, then the correlation matrix of the $a_{l, m}$ coefficients is diagonal: $\left\langle a_{l, m}\left(a_{l^{\prime}, m^{\prime}}\right)^{*}\right\rangle=C_{l} \delta_{l, l^{\prime}} \delta_{m, m^{\prime}}$. (Because of isotropy there is no dependence on $m$ ). Likewise, we find that $(\mathbf{C})_{i, j} \equiv\left\langle a_{i} a_{j}\right\rangle$ is diagonal in such a case. This fact makes the inversion of the covariance matrix in Eq. (1) trivial. Let us now relax the assumption on having $\boldsymbol{s}(\hat{\boldsymbol{n}})$ defined over the full sphere. In an astrophysical context, if some parts of the sky lack data, i.e., if $f_{\text {sky }}<1$, the covariance matrix $\mathbf{C}$ will no longer be diagonal and it will also be singular for large enough $l_{\max }$. A traditional matrix inversion is likely to either fail or provide inaccurate results. (The accuracy of the inversion can be tested by running Monte Carlo simulations and comparing the dispersion of the recovered $\hat{\alpha}$ 's with the actual prediction of Eq. (2)).

In these circumstances, we perform an SVD decomposition of the covariance matrix,

\section{$\mathbf{C}=\mathbf{R}^{\mathrm{t}} \boldsymbol{\Lambda} \mathbf{R}$,}

where $\boldsymbol{\Lambda}$ is a diagonal matrix (containing the eigenvalues of $\mathbf{C}$ ) and $\mathbf{R}$ a rotation orthogonal matrix $\left(\mathbf{R}^{\mathrm{t}} \mathbf{R}=\mathbf{I}\right)$. Since $\mathbf{C}$ is symmetric and positive definite, the eigenvalues should all be positive $^{3}$. The SVD decomposition sorts the eigenvectors according to the magnitude of the eigenvalues, so the first eigenvectors are those containing more information about $\mathbf{C}$, whereas the last ones are the most likely ones to introduce numerical noise. There is always some numerical error in our estimates of the covariance matrix, since it is computed through a finite number of Monte Carlo realizations (10000 in this work). Therefore, this decomposition provides a way to rotate the vector $\boldsymbol{a}$ into its principal modes, and permits distinguishing those having most of the information from those being more affected by numerical error (which can be safely projected out of the analysis). In practice, we neglected all eigenvectors whose eigenvalues were lower that a given fraction $\epsilon$ of the first (highest) eigenvalue. (For most cases, the choice $\epsilon=10^{-8}$ yielded optimal results). The inversion of $\mathbf{C}$ after the SVD decomposition becomes straightforward, enabling an easy implementation of the matched filter as given by Eq. (2). Unlike in Gorski (1994) or Mortlock et al. (2002), we are not worried about building a new set of orthonormal functions in the patch of the sky under analysis, nor do we attempt to perform component separation (Bouchet et al. 1999). In all those works, the techniques were in some way close to ours, but their goals were different.

As we see below, we may be interested in applying the matched filter in different $l$-bins. One can readily find that, given the outcome of the matched filter in two different $l$-bins $\hat{\alpha}_{p}$ and $\hat{\alpha}_{q}$, their covariance is given by

$$
(\tilde{\mathbf{C}})_{p, q} \equiv\left\langle\hat{\alpha}_{p} \hat{\alpha}_{q}\right\rangle-\left\langle\hat{\alpha}_{p}\right\rangle\left\langle\hat{\alpha}_{q}\right\rangle=\frac{\boldsymbol{m}_{p}^{\mathrm{t}} \mathbf{C}_{p p}^{-1} \mathbf{C}_{p q} \mathbf{C}_{q q}^{-1} \boldsymbol{m}_{q}}{\left(\boldsymbol{m}_{p}^{\mathrm{t}} \mathbf{C}_{p p}^{-1} \boldsymbol{m}_{p}\right)\left(\boldsymbol{m}_{q}^{\mathrm{t}} \mathbf{C}_{q q} \boldsymbol{m}_{q}\right)}
$$

\footnotetext{
3 In practice, we find that for dense and close to singular cases of $\mathbf{C}$, some eigenvalues (of a low absolute value) were negative.
} 
Here, $\mathbf{C}_{p p}$ and $\mathbf{C}_{q q}$ denote the covariance matrices of the noise $\boldsymbol{t}$ for $l$-bins $p$ and $q$, respectively, whereas $\mathbf{C}_{p q}$ is the covariance matrix for the noise in different $l$ bins $^{4}: \mathbf{C}_{p q}=\left\langle\boldsymbol{t}_{p} \boldsymbol{t}_{q}\right\rangle$. For a set of $l$-bins, we obtain a vector of measured $\hat{\alpha}_{p}$-s, whose combined $\chi^{2}$ is given by

$\chi^{2}[\hat{\alpha}]=\sum_{p, q} \hat{\alpha}_{p}\left(\tilde{\mathbf{C}}^{-\mathbf{1}}\right)_{p, q} \hat{\alpha}_{q}$.

An overall detection level for a given set of $l$-bins and $\hat{\alpha}$-s is provided by this $\chi^{2}$ statistic. Another statistic providing the level of detection is the variance-weighted average for the $\hat{\alpha}_{p}$ 's, here defined as $\hat{\beta}$ :

$\hat{\beta} \equiv \frac{\sum_{p}\left(\hat{\alpha}_{p} / \hat{\sigma}_{\alpha_{p}}^{2}\right)}{\sum_{p} 1 / \hat{\sigma}_{\alpha_{p}}^{2}}$.

We show below that, in ISW studies, the distribution of the $\hat{\alpha}_{p}$-S is very close to Gaussian, and so Gaussian is the distribution of $\hat{\beta}$. (The matched filter method, as defined from a minimization of the statistic given in Eq. (1), is only optimal if the noise is actually Gaussian-distributed). The diagonal elements of the matrix $\tilde{\mathbf{C}}$ can be computed via Eq. (9) or via numerical simulations: the agreement is very good (down to a few percent, compatible with the number of realizations). However, this agreement is not satisfactory for the non-diagonal elements when working under aggressive masks. In this case (and also when computing the dispersion of the statistic $\hat{\beta}$ ) we use the results obtained from 10000 Monte Carlo simulations. This assures a fair estimation of the correlation between different $\alpha_{p}$ estimates, hence an accurate estimation of the overall $\chi^{2}$ statistic.

\section{Comparison to the angular cross power spectrum}

In this section we shall compare the matched filter (as defined above) to the angular cross power spectrum (hereafter ACPS) method. This comparison is made within the model motivated in the previous section,

$s=t+\alpha \boldsymbol{m}$

and restricted to the large angular scales. This choice is motivated by the study of the ISW effect that follows in subsequent sections of the paper and that is typically restricted to $l<50$. When studying small scales, one has to be careful with the SVD decomposition, which might fail for the matrices that are too large. The matched filter in real space is, on most of those occasions, more adequate.

The ACPS can be viewed as a simplification of the matched filter presented here, where the covariance matrix is approximated by a diagonal matrix with identical non zero elements. Following the notation of Sect. 2.1, the estimate of $\alpha$ provided by this method is given by

$\hat{\alpha}_{\mathrm{ACPS}}=\frac{\sum_{l, m} m_{l, m}\left(s_{l, m}\right)^{*}}{\sum_{l, m}\left|m_{l, m}\right|^{2}}$

where $s_{l, m}$ and $m_{l, m}$ are the Fourier multipoles of the signals $s$ and $\boldsymbol{m}$, respectively. We refer to these signals in Fourier space,

\footnotetext{
4 According to our notation, $(\boldsymbol{a})_{i}=a_{i}$ denotes the $i$ th component of the array $\boldsymbol{a}$, where $\boldsymbol{a}_{p}$ denotes the $p$ th array of some larger group of arrays. Same for matrices: $(\mathbf{C})_{i, j}$ denotes the array element in the $i$ th row and $j$-th column, not to be confused with $\mathbf{C}_{p q}$, which denotes the covariance matrix computed from arrays $\boldsymbol{a}_{p}$ and $\boldsymbol{a}_{q}$.
}

and thus the vectors $\boldsymbol{s}$ and $\boldsymbol{m}$ will contain the components of the $s_{l, m}$ and $m_{l, m}$ multipoles as explained in Sect. 2.2.

To compare this method to the matched filter, we have to define $\boldsymbol{t}$ and $\boldsymbol{m}$ and build $\boldsymbol{s}$ according to Eq. (12). Throughout this paper, we do not use real data but only Gaussian realizations generated from a given cosmological model. For $\boldsymbol{t}$, we choose CMB realizations for which the ISW contribution has been subtracted; i.e., we simulate the Fourier multipoles $t_{l, m}$-s from an angular power spectrum computed using a modified version of the CMBFAST code (Seljak \& Zaldarriaga 1996) with a cosmological parameter set equal to the one given in Spergel et al. (2007): $\Omega_{c}=0.1994, \Omega_{\Lambda}=0.759, \Omega_{b}=0.0416, n_{s}=0.958$, $\sigma_{8}=0.75$, and $\tau=0.089$. This is the reference cosmological model hereafter. The template $\boldsymbol{m}$ is a Gaussian realization of a projection of the linear density field as computed from the matter power spectrum obtained with the same cosmological parameters. This density field is placed within a shell centered at $z=0.8$ with a total width of $\Delta z \sim 0.8$, i.e., the redshift range where ISW contribution is maximal (this will be addressed in detail in Sect. 4.1). To each realization of $\boldsymbol{t}$, we added the component $\alpha \boldsymbol{m}$ (for a given choice of $\alpha, \alpha=10^{-3}$ ), and applied the two methods. All maps were convolved with a Gaussian PSF of $F W H M=2^{\circ}$. Let us point out that by this exercise we do not attempt to simulate ISW observations, but simply to test the two methods in the context of Eq. (12).

In this comparison, we applied both the matched filter and the ACPS under two different masks: the first one assumes full sky coverage $\left(f_{\text {sky }}=1\right)$, whereas the second one adopts the mask provided by the fourth data release of Sloan Digital Sky Survey (SDSS-DR4, Eisenstein et al. 2001) combined with the Kp0 mask used in WMAP data analyses, (Hinshaw et al. 2003, see left panel of Fig. 1). In Table 1 we display the results after applying both methods to an ensemble of 10000 simulations. We consider two scenarios: (i) a unique $l$-bin limited to $l_{\max }=15$, $l \in[2,15]$; and (ii) a set of $15 l$-bins, ranging from $l_{\min }=2$ to $l_{\max }=50: l \in[2,3],[4,5],[6,8],[9,14],[15,25],[26,28],[29,31]$, $[32,34],[35,37],[38,40],[41,43],[44,45],[46,47],[48,49]$ and [50,51]. We quote the results for the $\hat{\alpha}$ statistic in case (i), and for the $\chi^{2}$ and $\hat{\beta}$ statistics in case (ii). Note that, in this case, the $\chi^{2}$ statistic has been normalized by the number of degrees of freedom, i.e., the number of $l$-bins.

We always find that the estimates of $\tilde{\alpha}$ are unbiased for both methods ${ }^{5}$ and that the matched filter provides an estimate of the dispersion of $\tilde{\alpha}$ (see Eq. (2)) that actually agrees with the value recovered from the Monte Carlo simulations. In case (i) we obtain that the matched filter works better than the ACPS under the two masks considered. The noise signal in these analyses corresponds to the CMB (after having the ISW component subtracted), so the noise power spectrum scales as $\left\langle\left|t_{l, m}\right|^{2}\right\rangle \propto l^{-2}$. On the other hand, the angular power spectrum of our density template scales roughly as $\left\langle\left|m_{l, m}\right|^{2}\right\rangle \propto$ const. at $l<50$. This means that for the low- $l$ range considered in (i), the matched filter is going to weight the high- $l$ end multipoles more. This effect makes this method superior to the ACPS (which weights all multipoles equally) in the all-sky case. For the SDSS-DR4 mask, the matched filter also accounts for the coupling among mutipoles, and this enlarges the difference between the two methods. One obvious question that arises is how the matched filter can

\footnotetext{
5 A mask in real space involves a convolution of different $\alpha$ values in Fourier space, which may generate a bias if $\alpha$ is not constant versus $l$. The case considered in this section observes a constant $\alpha$ for every multipole, but in subsequent sections this will not be the case and a bias will appear.
} 

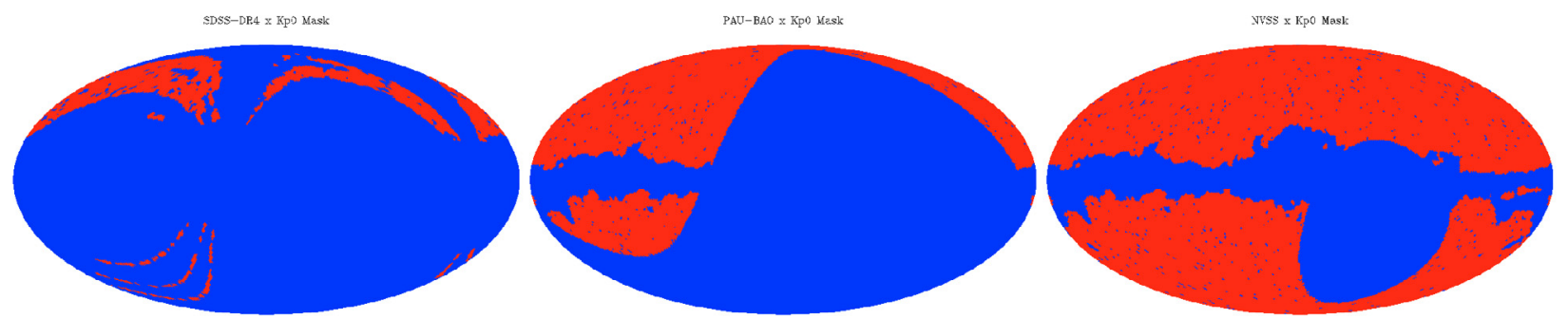

Fig. 1. Three different masks used in this paper. Left: mask corresponding to the product of the SDSS-DR4 mask times the Kp0 mask used in Hinshaw et al. (2003). Middle: mask assigned to the future PAU-BAO galaxy survey times the Kp0 mask. Right: mask of the NVSS times the Kp0 mask.

Table 1. Comparison of the matched filter (MF) and the ACPS under two different masks (SDSS-DR4 mask and all sky).

\begin{tabular}{c|cc|cc|cc}
\hline \hline & \multicolumn{2}{|c|}{$l_{\max }=15$} & \multicolumn{4}{c}{$l_{\max }=50$} \\
\hline & MF & ACPS & \multicolumn{2}{|c|}{ MF } & \multicolumn{2}{c}{ ACPS } \\
\hline & \multicolumn{2}{|c|}{$\langle\hat{\alpha}\rangle / \sigma_{\hat{\alpha}}$} & $\left\langle\chi_{N}^{2}\right\rangle$ & $\langle\hat{\beta}\rangle / \sigma_{\hat{\beta}}$ & $\left\langle\chi_{N}^{2}\right\rangle$ & $\langle\hat{\beta}\rangle / \sigma_{\hat{\beta}}$ \\
\hline All sky & 25.70 & 16.50 & 2,312 & 192 & 2,316 & 192 \\
SDSS DR4 & 34.68 & 6.44 & 271 & 63 & 201 & 55 \\
\hline
\end{tabular}

perform better under the SDSS-DR4 mask than in the all sky case. The low $l$ modes are degenerate under the SDSS-DR4 mask, that is, they are not orthonormal as for $f_{\text {sky }}=1$ and are decomposed onto other modes corresponding to smaller angular scales. That means that the noisiest (low $l$ ) modes under the full sky mask are not eigenmodes anymore and they are partially dropped from the analysis (the matched filter method handles 176 different modes under the SDSS-DR4 mask, as opposed to 252 modes if $f_{\text {sky }}=1$ ). This means that under the SDSS-DR4 mask we have a different statistic (since it handles a different number of degrees of freedom) that is more concentrated on angular scales where the noise amplitude is smaller. This provides this new statistic a better $S / N$.

In case (ii) and $f_{\text {sky }}=1$, it turns out that, given the scaling of the power spectra of $\boldsymbol{m}$ and $\boldsymbol{t}$, most of the $S / N$ is in the few higher $l$ bins, centered on multipoles $l=45,46, \ldots, 50$. Indeed, these last multipoles dominate the sums in Eq. (13). For these few high- $l$ bins carrying most of the information, the change in the noise properties is very small, so each of these bins is roughly equivalent to the rest. The weighting applied by the matched filter introduces very little difference, and both methods perform similarly, yielding almost identical values of $\chi^{2}$. But again, under the SDSS-DR4 mask the coupling among multipoles is observed by the matched filter, which introduces a difference between the two methods. However, it is clear that the matched filter performs better when a single $l$-bin is considered (case (i)).

\section{Application to ISW studies}

The ISW effect arises as a consequence of a late time variation in the gravitational potentials on the large scales. If there is a net change in the depth of the potential wells while they are being crossed by $\mathrm{CMB}$ photons, then this radiation field will experience a gravitational red/blueshift. Crittenden \& Turok (1996) point out that gravitational potentials should be traced by the large scale structure (LSS), and proposed the cross-correlation of CMB maps to LSS surveys to unveil this signal. However, in most plausible models, the time variation of the potentials occurs at late times (or low redshifts, $z<2$ ), and the angle subtended by the linear scales for which the ISW effect is important is rather large $\left(\theta>3-5^{\circ}\right)$. This means that there will only be room for relatively few independent ISW spots on the sky; i.e., this effect will be considerably limited by cosmic variance. Further, it is in this large angular range where the Galactic emission is more important, and errors in its subtraction might be more relevant. For this reason, it becomes necessary to use masks that project out regions where this galactic contamination is large and cannot be removed accurately. Furthermore, when doing a crosscorrelation analysis between CMB maps and LSS maps, the latter may not cover the whole sky, but will also be restricted to a given limited region. In this context, it becomes particularly important to implement cross-correlation tools that are as sensitive as possible and able to optimally handle the limitations imposed by the sky masks.

\subsection{The $S / N$ in ISW studies}

In this section we briefly describe the ISW-LSS crosscorrelation in the frame of the WMAP3 cosmogony. Unlike Douspis et al. (2008), we refrain from addressing the dependence of this correlation with different dark energy models. We concentrate on a single LSS survey, and search for its optimal redshift in terms of ISW detection. We study the amount of $S / N$ that arises in those cases, and the angular scales where it is generated. This sets the scenario for our cross-correlation method comparison.

The ISW-LSS cross correlation arises from both LSS probes and gravitational potentials tracing the underlying matter density field. The expression for the temperature anisotropies introduced by a gravitational blue/redshift reads as (Sachs \& Wolfe 1967; Martinez-Gonzalez et al. 1990)

$\left(\frac{\delta T}{T_{0}}\right)_{\text {ISW }}(\hat{\boldsymbol{n}})=\frac{-2}{c^{3}} \int \mathrm{d} t \dot{\phi}(\hat{\boldsymbol{n}}, t)$.

The symbol $\dot{\phi}$ denotes the time derivative of the gravitational potential $\phi$. In linear theory, this expression can be rewritten as (e.g., Cooray 2002):

$$
\begin{aligned}
a_{l, m}^{\mathrm{ISW}}=(-i)^{l}(4 \pi) \int \frac{\mathrm{d} \boldsymbol{k}}{(2 \pi)^{3}} Y_{l, m}^{\star}(\hat{\boldsymbol{k}}) \\
\quad \times \int \mathrm{d} r j_{l}(k r) \frac{-3 \Omega_{m} H_{0}^{2}}{k^{2}} \frac{\mathrm{d}(D / a)}{\mathrm{d} r} \delta_{\boldsymbol{k}} .
\end{aligned}
$$

The multipole coefficients $a_{l, m}^{\mathrm{ISW}}$ are related to the ISW temperature anisotropies by Eq. (3). In this equation, $r$ denotes comoving distance, $k$ comoving wavevector, $j_{l}(x)$ denotes the spherical Bessel function of order $l, H_{0}$ is the Hubble parameter, $a(r)$ the scale factor, and $D(r)$ the standard linear growth factor. The $3 \mathrm{D}$ Fourier mode of the density contrast is denoted by $\delta_{k}$. For an Einstein-de Sitter universe $(D=a)$, the whole integral vanishes. 
In a similar way, the multipole coefficients for the angular number density of a matter density probe (which will be taken to be galaxies in what follows) read as

$$
\begin{aligned}
a_{l, m}^{\mathrm{g}}=(-i)^{l}(4 \pi) & \int \frac{\mathrm{d} \boldsymbol{k}}{(2 \pi)^{3}} Y_{l, m}^{\star}(\hat{\boldsymbol{k}}) \\
& \times \int \mathrm{d} r j_{l}(k r) r^{2} n_{g}(r) b(r, k) D(r) \delta_{\boldsymbol{k}},
\end{aligned}
$$

with $n_{\mathrm{g}}(r)$ the average number density of galaxies. The bias function $b(r, k)$ accounts for the usual probes of the LSS actually being biased tracers of the underlying mass distribution $(b>1)$. This expression neglects the presence of shot (Poisson) noise in the galaxy number. The coordinate $r$ here is taken as a lookback time coordinate, equivalent to conformal time or redshift (z). For the sake of clarity, in what follows we use $z$ as the lookback time coordinate. We find that a given galaxy survey will probe the redshift range given by the product $\Pi(z) \equiv b N_{\mathrm{g}}$, with $N_{\mathrm{g}}(z) \equiv r^{2}(z) n_{\mathrm{g}}(z) D(z)$. At this stage, we are ignoring the $k$ dependence of the bias function $b$. For simplicity, we rewrite Eqs. (15-16) as

$a_{l, m}^{\mathrm{ISW}, \mathrm{g}}=(-i)^{l}(4 \pi) \int \frac{\mathrm{d} \boldsymbol{k}}{(2 \pi)^{3}} Y_{l, m}^{\star}(\hat{\boldsymbol{k}}) \times \Delta_{l}^{\mathrm{ISW}, \mathrm{g}}(k, z)$,

with the $\Delta_{l}^{\mathrm{ISW}, \mathrm{g}}(k, z)$ referred to as transfer functions of the ISW and the galaxy fields, respectively. In real space, the cross correlation function between ISW temperature anisotropies and LSS probes reads as

$C_{\mathrm{ISW} \otimes \mathrm{g}}(\theta)=\sum_{l} \frac{2 l+1}{4 \pi} C_{l}^{\mathrm{ISW} \otimes \mathrm{g}} P_{l}(\cos \theta)$,

with $C_{l}^{\mathrm{ISW} \otimes \mathrm{g}}$ the cross power spectrum,

$C_{l}^{\mathrm{ISW} \otimes \mathrm{g}}=\left(\frac{2}{\pi}\right) \int k^{2} \mathrm{~d} k \Delta_{l}^{\mathrm{ISW}} \Delta_{l}^{\mathrm{g}} P_{m}(k)$,

and $P_{m}(k)$ the linear matter power spectrum. The symbol " $\otimes$ " denotes cross correlation. The theory makes actual predictions for this cross-power spectrum, and its covariance matrix is diagonal if $f_{\text {sky }}=1$ and, in general, is simpler than for correlation function, (see the detailed analysis of Cabré et al. 2007). As an example, we show a cross power spectrum in the top panel of Fig. 2. For the sake of comparison, we also display the total $\mathrm{CMB}$ contribution (for the chosen $\Lambda \mathrm{CDM}$ model) and its ISW component. The angular power spectrum corresponding to the projection of the galaxy field whose window function $\Pi(z)$ is centered at $z=0.8$ is displayed in that plot too. The total width in redshift space is roughly $\Delta z \approx 0.80$, so it is a thick shell. The cross power spectrum peaks at scales at around $l \sim 30-50$, but its amplitude at $l \sim 200$ is roughly equal to that at $l \sim 2$. This might suggest that the there is so much cross-correlation signal at large $(l<10)$ as on small $(l>100)$ scales, but indeed most $(90 \%)$ of the signal comes from the large scales $(l<40)$ if $f_{\text {sky }}=1$, as we show below. The amplitude of the density power spectrum (and hence the cross power spectrum) are taken as arbitrary. The $S / N$ for the measurement of a given multipole $l$ of the cross power spectrum can be computed once one takes into account that the $a_{l, 0}$ multipoles are defined real and that the real and complex components of the $a_{l, m}(m>0)$ multipoles, besides being equivalent and independent, must satisfy the constraint for the total amplitude $\left\langle\left|a_{l, m}\right|^{2}\right\rangle=C_{l}$. We obtain

$$
\left(\frac{S}{N}\right)_{l}^{2}=\frac{f_{\mathrm{sky}}\left(C_{l}^{\mathrm{ISW} \otimes \mathrm{g}}\right)^{2}(l+1)^{2}}{\left[C_{l}^{\mathrm{CMB}} C_{l}^{\mathrm{g}}+\left(C_{l}^{\mathrm{ISW} \otimes \mathrm{g}}\right)^{2}\right](l / 2+1)} .
$$
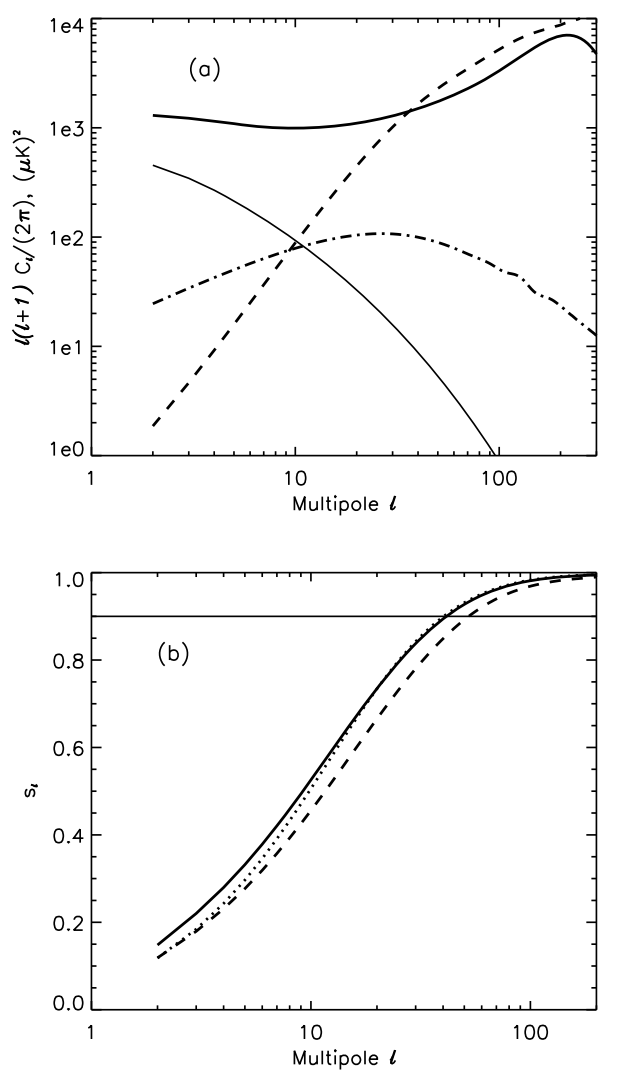

Fig. 2. a) The dot-dashed line displays the cross power spectrum of the projected density field through a shell centered at $z=0.8$ (dashed line) times the ISW component (thin solid line). The thick solid line displays the total CMB angular power spectrum. b) Normalized $S / N$ (as given by Eq. (21)) versus multipole $l$ : the solid line corresponds to a density probe in a shell centered at $z=0.8$, whereas for the dotted and dashed lines the central redshifts are 0.4 and 1.3 , respectively. It is assumed that $f_{\text {sky }}=1$. In both panels, the width of each density shell is equal to $20 \%$ of the comoving distance to the central-shell redshift.

In this equation, $C_{l}^{\mathrm{CMB}}$ is the $\mathrm{CMB}$ angular power spectrum and $C_{l}^{\mathrm{g}}$ is the LSS probe auto power spectrum. The quantity

$$
s_{l} \equiv \sqrt{\frac{\sum_{l^{\prime}=2}^{l^{\prime}=l}(S / N)_{l^{\prime}}^{2}}{\sum_{l^{\prime}=2}^{l^{\prime}=l_{\max }}(S / N)_{l^{\prime}}^{2}}}
$$

is displayed versus $l$ in the bottom panel of Fig. 2 ; i.e., this figure shows the ratio of the $S / N$ contained below some given $l$ for shells of different widths placed at different redshifts. We see that, regardless of where $\Pi(z)$ peaks, practically half of the total signal is contained at multipoles $l<10$, whereas its $90 \%$ fraction is typically contained at $l<40-50$. (Had we considered thinner shells - width equal to $2 \%$ of the comoving distance-, then all those shells below $z=0.8$ would have still shown a pattern very close to that given by the solid line). This suggests that by dropping all multipoles above $l=50$ (or by neglecting scales smaller than $\theta \approx 3^{\circ}-4^{\circ}$, , one should recover practically the same ISW detection significance. This sets a useful consistency check, given the number of other physical phenomena (Rees-Sciama effect (Rees \& Sciama 1968), kinetic Sunyaev-Zel'dovich effect (Sunyaev \& Zeldovich 1972), and intrinsic source emission, etc.) that arise on smaller angular scales and that, a priori, correlate with the spatial position of LSS probes.

In Fig. 3 we show how the total $S / N$ depends on the width and on the central redshift for the galaxy survey window function 


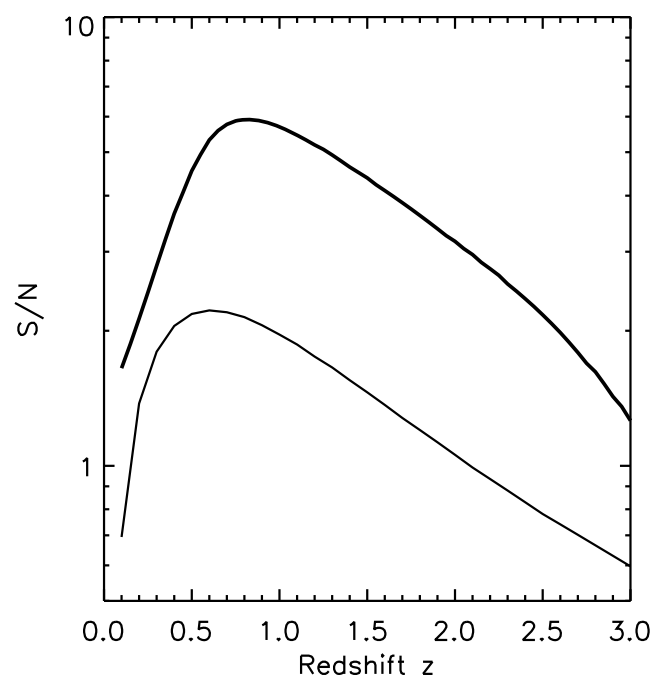

Fig. 3. Total $S / N$ of the cross power spectrum detection as given by the numerator of Eq. (21) with respect to the central redshift of the density probe shell. The thick solid line displays the case when the shell containing the density probes has a width equal to $20 \%$ of the comoving distance to the shell. For the thin solid line, this width is only $2 \%$.

$\Pi(z)$. In the thick shell case, the width is taken to be roughly $20 \%$ of the comoving distance to the central redshift. In redshift space, it implies a width of $\Delta z \approx 0.7$ for central redshift $z=0.3$, $\Delta z \approx 0.84$ for central redshift $z=0.8$, and $\Delta z \approx 0.99$ for central redshift $z=1.3$. The thin shell case observes a width of only $2 \%$ the comoving distance to the central redshift, and this translates into $\Delta z \approx 0.07,0.08$, and 0.1 for central redshifts $z=0.3,0.8$, and 1.3 , respectively. We should obtain the larger detection levels for thick shells (large $\Delta z$ 's) and $z=0.8$, which motivates our choice of a thick survey centered at this redshift ${ }^{6}$. Our assumption that the bias is independent of scale might not be accurate, but it has less impact on the large scales (low $l \mathrm{~s}$ ) where most of the effect is coming from. We do not expect significant changes after introducing a scale-dependent bias in our galaxy survey description, although we address this issue in detail when applying our method to real CMB and LSS data. Note that, a priori, this method can be applied similarly on multiple redshift shells and is affected in exactly the same way as the ACPS by realistic aspects, such as the redshift or scale dependence of the bias, survey incompleteness, etc.

We next compare the performance of the matched filter to that of the ACPS. We use one Gaussian realization of our chosen density shell, and compute a single Gaussian realization of an ISW map compatible with it. If $a_{l, m}^{\mathrm{g}}$ are the Fourier multipoles of our density 2D template, then they can be related to those of a compatible ISW map via (e.g., Cabré et al. 2007):

$a_{l, m}^{\mathrm{ISW}}=\alpha_{l} a_{l, m}^{\mathrm{g}}+\beta_{l, m}=\frac{C_{l}^{\mathrm{ISW} \otimes \mathrm{g}}}{C_{l}^{\mathrm{g}}} a_{l, m}^{\mathrm{g}}+\beta_{l, m}$.

The Gaussian signal $\beta_{l, m}$ is the part of the ISW component that is uncorrelated to the LSS $2 \mathrm{D}$ template, verifying $\left\langle\left|\beta_{l, m}\right|^{2}\right\rangle=$ $C_{l}^{\mathrm{ISW}}-\left(C_{l}^{\mathrm{ISW} \otimes \mathrm{g}}\right)^{2} / C_{l}^{\mathrm{g}}$, where $C_{l}^{\mathrm{g}}$ denotes the angular power spectrum of the LSS probe. This is the correct way to express the ISW field in terms of the galaxy density field, as long as both

${ }^{6}$ It has been noted elsewhere (e.g. Afshordi 2004) that, by combining different LSS surveys at different redshifts, one can obtain larger $S / N$. We avoid that discussion here and focus our method comparison on one single survey. fields are Gaussian and they are completely determined by the first and second order momenta. The ratio $C_{l}^{\mathrm{ISW} \otimes \mathrm{g}} / C_{l}^{\mathrm{g}}$ is explicitly identified with $\alpha_{l}$, which is precisely the output of our matched-filter technique. According to the theory, $\alpha_{l}$ shows a strong dependence on $l$, meaning our method must be applied in separate $l$-bins. Gaussian simulations of the CMB were built from the addition of our fixed ISW template plus realizations of a CMB angular power spectrum for which the ISW component had been subtracted, just as for the $t$ component in Sect. 3. The realizations from the modified $\mathrm{CMB}$ angular power spectrum were computed up to a maximum multipole $l=160$, and convolved with a Gaussian beam of $2^{\circ}$ of FWHM. The fixed ISW and the LSS maps were also convolved with the same PSF, and all maps were produced under the HEALPix (Górski et al. 2005) ${ }^{7}$ resolution parameter $N_{\text {side }}=64$.

\subsection{Performance under different masks}

The simulated maps were cross-correlated to the projected density map (hereafter denoted as $\boldsymbol{m}$ ) with both the ACPS and the matched-filter methods, according to the multipole decomposition given in Sect. 2.2. Their performance was compared under three different masks shown in Fig. 1: the left panel shows the mask corresponding to the sky coverage of the fourth data release of the Sloan Digital Sky Survey (SDSS-DR4, Eisenstein et al. 2001). This mask was multiplied by the Kp0 mask used in the analysis of WMAP data (Hinshaw et al. 2003), so the combined mask observes a bit less than $10 \%$ of the total sky. In the middle panel we consider the fraction of the sky covered by the upcoming survey $\mathrm{PAU}_{-\mathrm{BAO}}{ }^{8}$. This survey is planned to cover $\sim 10000$ square degrees of the celestial northern hemisphere, and we have assumed that it is limited to the region $b>20^{\circ}$ outside the $\mathrm{Kp} 0$ mask, in such a way that $f_{\text {sky }} \simeq 0.26$. Finally, the righthand side panel displays the product of the Kp0 mask with the mask corresponding to the NVSS survey (Condon et al. 1998). In this case, $f_{\text {sky }} \simeq 0.65$.

A total of 10000 simulations were run for the method comparison, and results are given in Table 2 . The sensitivity of both methods is measured by the $\chi^{2}$ and the $\hat{\beta}$ statistics for different choices of the maximum multipole $l_{\max }$ considered in the analyses. In each case, the $\chi^{2}$ statistic has been normalized by the number of degrees of freedom, i.e., the number of $l$-bins. In total, we considered $20 l$-bins: $l \in[2,3],[4,5],[6,8],[9,14],[15,25]$, $[26,28],[29,31],[32,34],[35,37],[38,40],[41,43],[44,45]$, $[46,47],[48,49],[50,51],[52,53],[54,55],[56,57],[58,59]$ and $[60,61]$. We see that, unlike in the previous section (where the matched filter was in general significantly more sensitive under the data model $\boldsymbol{s}=\boldsymbol{t}+\alpha \boldsymbol{m})$, both filters perform very similarly in this ISW context. There seems to be, however, a slighter better sensitivity of the matched filter under the most aggressive masks, but the difference is small (at least in terms of the output of the $\chi^{2}$ and $\hat{\beta}$ statistics). We note that the ACPS is the Legendre transform of the angular cross correlation function and that both methods should, a priori, show similar sensitivities. Of the two statistics considered in Table $2, \hat{\beta}$ provides the most significant ISW detections (its distribution is very close to Gaussian, and the number of sigmas yield lower chance probabilities). It is remarkable that for both of them the ISW detection significance finds a maximum at $l \sim 5-40$, and then starts dropping again. According to this result, by merely observing multipoles below $l=40$,

\footnotetext{
7 http://www.healpix.jpl.nasa.gov

8 http://www.ice.csic.es/research/PAU/

PAU-welcome.html
} 
Table 2. Comparison of the matched filter (MF) to the ACPS in the context of ISW studies.

\begin{tabular}{c|c|cc|cc|cc|cc|cc}
\hline \hline & & \multicolumn{2}{|c|}{$l_{\max }=5$} & \multicolumn{2}{c|}{$l_{\max }=14$} & \multicolumn{2}{c|}{$l_{\max }=31$} & \multicolumn{2}{c|}{$l_{\max }=40$} & \multicolumn{2}{c}{$l_{\max }=51$} \\
\hline & & MF & ACPS & MF & ACPS & MF & ACPS & MF & ACPS & MF & ACPS \\
\hline \multirow{2}{*}{ SDSS-DR4 } & $\left\langle\chi_{N}^{2}\right\rangle$ & 1.79 & 1.31 & 2.04 & 1.56 & 1.61 & 1.48 & 1.46 & 1.40 & 1.33 & 1.30 \\
& $\langle\hat{\beta}\rangle / \sigma_{\hat{\beta}}$ & 1.24 & 0.65 & 1.87 & 1.35 & 1.74 & 1.70 & 1.57 & 1.61 & 1.46 & 1.22 \\
PAU-BAO & $\left\langle\chi_{N}^{2}\right\rangle$ & 2.93 & 2.02 & 2.94 & 2.45 & 2.12 & 2.29 & 1.87 & 2.02 & 1.61 & 1.74 \\
& $\langle\hat{\beta}\rangle / \sigma_{\hat{\beta}}$ & 1.69 & 1.28 & 2.48 & 2.22 & 1.93 & 2.33 & 1.90 & 2.33 & 1.69 & 1.98 \\
\multirow{3}{*}{ NVSS } & $\left\langle\chi_{N}^{2}\right\rangle$ & 6.27 & 7.03 & 5.30 & 6.04 & 4.15 & 4.71 & 3.47 & 3.91 & 2.75 & 3.06 \\
& $\langle\hat{\beta}\rangle / \sigma_{\hat{\beta}}$ & 3.24 & 3.37 & 3.51 & 3.70 & 3.44 & 3.62 & 3.43 & 3.56 & 3.19 & 3.19 \\
\hline
\end{tabular}
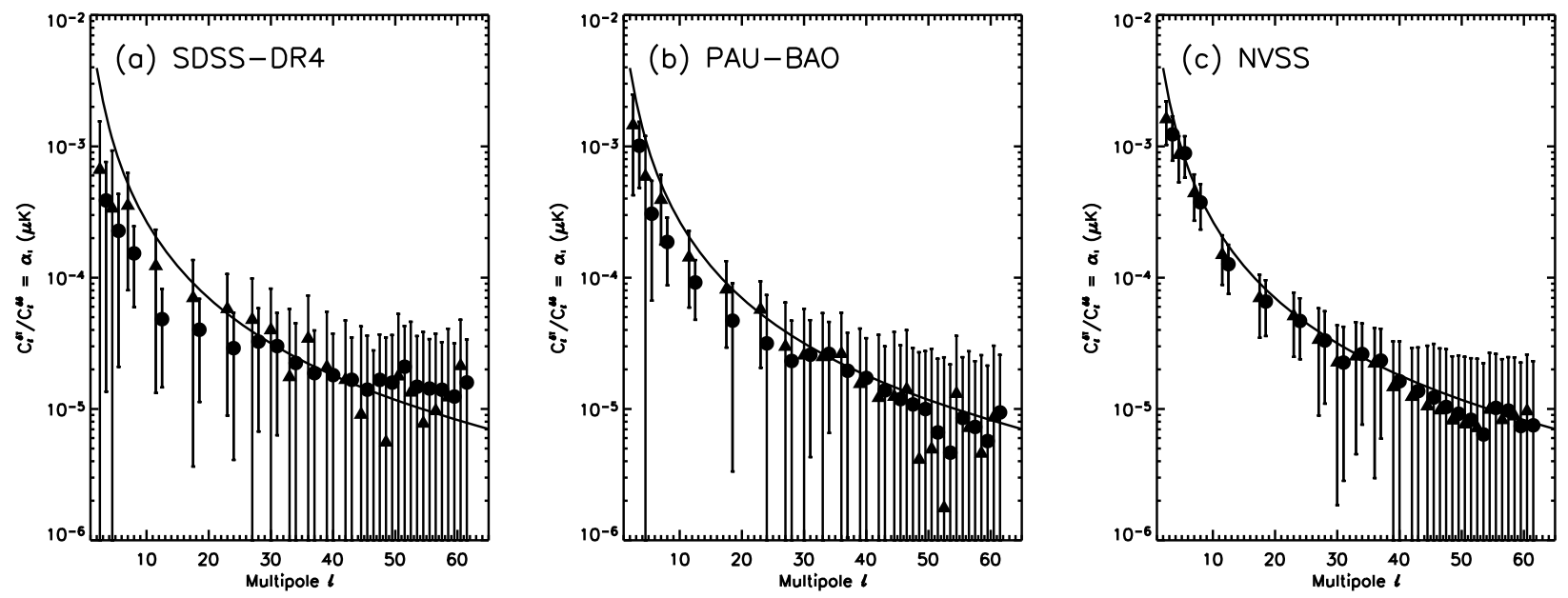

Fig. 4. Recovered values of $\alpha_{l}=C_{l}^{\mathrm{ISW} \otimes \mathrm{g}} / C_{l}^{\mathrm{g}}$ with the matched filter (filled circles) and the ACPS (filled triangles) under the three masks considered: a) SDSS-DR $4 \times \mathrm{Kp} 0$, b) PAU-BAO $\times \mathrm{Kp} 0$, and c) NVSS $\times \mathrm{Kp} 0$. Error bars denote the rms scatter for each of them. Solid lines display the theoretical prediction for the correlation coefficient $\alpha_{l}=C_{l}^{\mathrm{ISW} \otimes g} / C_{l}^{\mathrm{g}}$ versus multipole $l$. Our choice for the density and ISW templates is such that full sky analyses should yield $\alpha_{l}$ estimates exactly on the solid lines.

we should recover practically all the ISW-LSS cross correlation significance. This points in the direction of our $s_{l}$ estimates in Sect. 4.1, which predicted most of the $S / N$ will be confined at low $l$-s and should prevent confusion with other secondary effects (giving rise to correlations of other nature, such as the Rees-Sciama effect and the kinetic Sunyaev-Zel'dovich effect).

One advantage of the correlation methods implemented here is that they conduct analyses in separate ranges of angular scales, enabling a direct comparison with theoretical predictions. This is explicitly shown in Figs. 4 and 5. Let us first note that, according to the error bars displayed in Fig. 4, most of the information again seems to be restricted to the large angular scales $(l<30-40)$, as quoted above. Let us now address the issue of the impact of the mask on the methods' output. A clear low bias can be seen in the estimates of $\alpha_{l}$ for low $l$-s, especially under the SDSS-DR4 and PAU-BAO masks. This is a direct effect of the mask: a multiplication of the full sky map by the actual mask in real space translates into a convolution in Fourier space, which involves a wider range of multipoles when the mask is smaller. Therefore, the $\alpha_{l}$ estimates for the SDSS-DR4 mask will be the result of an average or smoothing of the $\alpha_{l}$ values in a wide space of $l$ multipoles. Since at low $l$-s, the values of $\alpha_{l}$ fall steeply, the convolution will provide a value that is lower than the actual theoretical value, as displayed in the left panel of Fig. 4.

At the same time, the mask introduces another bias in the high $l$ range, for which the actual recovered values of $\alpha_{l}$ are above the theoretical expectation. This shows that the aliasing introduced by the mask is shifting some large angle (low $l$ ) power into the small angle (high $l$ ) range. We attempted to quantify this aliasing by performing the following exercise. We generated one ISW map by using multipoles restricted to the range $l \in[2,10]$. We multiplied this map by the SDSS-DR4 mask used in this work and computed the power spectrum of the resulting map. We measured the aliased variance contained above some $l_{\min }>10$ by using $\sigma^{2}\left[l_{\min }\right]=\sum_{l_{\min }}(2 l+1) /(4 \pi) C_{l}$. We found that about $30 \%$ of the total $\mathrm{rms}^{9}$ was contained above $l_{\min }=30$; i.e., the ISW power aliasing from large to small scales is indeed significant. Let us note as well that this effect is more present for the matched filter $\alpha_{l}$ estimates, as we discuss next.

A direct visual comparison of the two methods can be found in Fig. 5, where the $S / N$ for each multipole bin is shown. The matched filter performs more accurately than the ACPS method, especially under the SDSS-DR4 and PAU-BAO masks, although this has a limited impact on the final detection significance quoted by the $\chi^{2}$ and $\hat{\beta}$ statistics (see next section).

\section{Discussion and conclusions}

In order to assess the sensitivity of the two methods to the ISW, we have defined two different statistics: the $\chi^{2}$ statistic uses a quadratic combination of the method's output (in a similar way as in Tegmark 1997), and the $\hat{\beta}$ statistic, which instead is linear in the $\hat{\alpha}_{l}$ 's and for our purposes is Gaussian-distributed. Both statistics pick up the information of the cross-correlation in different ways. The $\chi^{2}$ statistic is more sensitive to the presence

\footnotetext{
9 The total rms was computed by taking $l_{\min }=2$, and was less than $10 \%$ off the estimate obtained from the map in real space.
} 

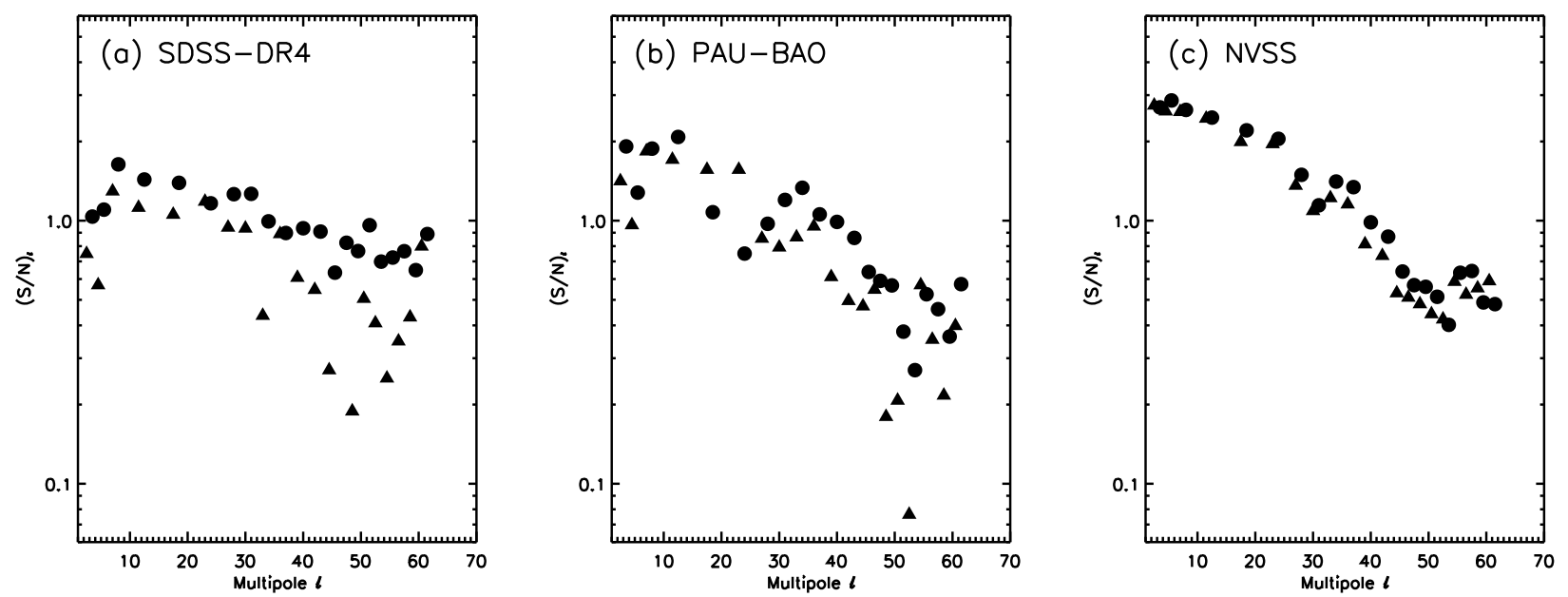

Fig. 5. Recovered $S / N$ for each multipole bin with the matched filter (filled circles) and the ACPS (filled triangles), under the three masks considered in Fig. 4.

of ISW only on the very large angular scales, and rapidly gets degraded as smaller angular scales are considered; i.e., it seems to be particularly affected by the inclusion of modes that have a low $S / N$. On the other hand, the $\hat{\beta}$ statistic is more sensitive to the actual $S / N$ even on scales where such ratio is below unity, and, as mentioned above, seems to be more efficient in terms of detection of the ISW-LSS cross-correlation.

This connects to the multipole or angular range where most of the correlation is arising. Of the two methods, the matched filter seems to be more confined in $l$-space than the ACPS when looking at the output of the $\hat{\beta}$ statistic. It quotes the maximum significance at $l_{\max }=14$ and always drops at larger $l_{\max }-\mathrm{s}$, whereas the ACPS seems to peak at around $l_{\max }=30-40$. In this case, the exception is the NVSS-like survey, for which $\hat{\beta}$ yields the maximum detection significance at $l_{\max }=14$. This different behavior suggests that aliasing induced by SDSS-DR4 and PAU-BAO masks is indeed shifting some $S / N$ into the smaller scales (larger $l$-s), but this effect is not perceptible beyond $l_{\max }=30-40$. This is also visible in Figs. 4 and 5: under the most aggressive masks, there is less information in the first $l$-bins $(l \in[2,3],[4,5])$, whereas these contain the highest values of the $S / N$ for the NVSS-like survey.

These two figures also show that, in almost every $l$-bin, the matched filter $\alpha_{l}$ estimates seem to be more accurate than the ACPS method and that, at the same time, they seem to be more affected by aliasing. These two facts are connected: the matched filter tends to pick up the signal from those modes having the highest $S / N$ within each $l$-bin. For small $f_{\text {sky }}$ and moderately high $l$-bins, these modes are actually aliased components of low $l$ modes whose power has been shifted by the mask onto smaller scales. This makes the matched filter provide more accurate $\alpha_{l}$ values in these high $l$-bins, but these estimates are actually highly correlated to those found at lower $l$-s. This limits the amount of information that high- $l$ bins actually add, and partially explains the high bias of the $\alpha_{l}$ estimates at large $l$-s in the left panel of Fig. 4.

We generalized the implementation of the matched filter into the Fourier space of the 2D sphere and applied it in the context of CMB analyses and ISW studies. The matched filter provides a tool for estimating the level of presence of some template $\boldsymbol{m}$ in some measured signal $s$ containing a noise component $t$. This tool is optimized when $\boldsymbol{t}$ is isotropic and Gaussian-distributed, and hence is particularly suited to cross-correlation tests where the CMB is the background (noise) signal. In Fourier (or multipole) space, the correlation properties of the CMB are particularly simple (especially, but not only, in the full sky case $\left.f_{\text {sky }}=1\right)$. For $f_{\text {sky }}<1$, the covariance matrix of the CMB multipoles can be inverted via an SVD approach, which permits simultaneously identifying those Fourier modes containing more information and dropping the other modes that introduce numerical error. After all modes have been sorted in terms of their $S / N$, the matched filter algorithm weights them accordingly to produce an optimal (minimum variance) output for the cross-correlation test. We compared this method with the standard ACPS, and found the the matched-filter to be either superior or equivalent to the ACPS.

In the context of ISW analyses, the matched filter estimates the level of cross-correlation of CMB maps with LSS probes at separate multipole ranges, and this enables a direct and clean comparison to theoretical predictions. When applying both the matched filter and the ACPS methods to three mock surveys with distinct values of $f_{\text {sky }}$, we find that both methods perform similarly. The matched filter is slightly more sensitive under aggressive masks, the ACPS more accurate under the NVSS mask. The masks introduce some power aliasing from large onto small angular scales, but this does not prevent most of the $S / N$ of the ISW-LSS cross correlation from being confined into the large angular scales $(l<40)$. This $l$-confinement may be particularly useful when distinguishing this effect from other secondary anisotropies that, while tracing the LSS distribution, arise on smaller angular scales.

Natural extensions of this work involve large-angle component separation in future CMB maps when tracers or templates for the signal to be distinguished are available, such as galactic or extragalactic radio, synchrotron or dust maps, large-scale $1 / f$ noise component, local kSZ, or local tSZ contributions.

Acknowledgements. I am grateful to Tony Banday for carefully reading the manuscript. I acknowledge the use of the HEALPix (Górski et al. 2005) package and the LAMBDA ${ }^{10}$ data base.

${ }_{10}$ http://lambda.gsfc.nasa.gov 


\section{References}

Afshordi, N. 2004, PhRvD, 70, 083536

Atrio-Barandela, F., Kashlinsky, A., \& Mücket, J. P. 2004, ApJ, 601, L111

Atrio-Barandela, F., Mücket, J. P., \& Génova-Santos, R. 2008, ApJ, 674, L61

Battistelli, E. S., et al. 2006, [arXiv: astro-ph/0603702]

Bennett, C. L., Banday, A. J., Gorski, K. M., et al. 1996, ApJ, 464, L1

Bennett, C. L., Halpern, M., Hinshaw, G., et al. 2003, ApJS, 148, 1

Benson, B. A., Church, S. E., Ade, P. A. R., et al. 2003, ApJ, 592, 674

Bhattacharya, S., \& Kosowsky, A. 2007a, [arXiv:0712.0034]

Bhattacharya, S., \& Kosowsky, A. 2007b, ApJ, 659, L83

Böhringer, H., Voges, W., Huchra, J. P., et al. 2000, ApJS, 129, 435

Bouchet, F. R., Prunet, S., \& Sethi, S. K. 1999, MNRAS, 302, 663

Cabré, A., Fosalba, P., Gaztañaga, E., \& Manera, M. 2007, MNRAS, 381, 1347

Cayón, L., Sanz, J. L., Barreiro, R. B., et al. 2000, MNRAS, 315, 757

Cen, R., \& Ostriker, J. P. 1999, ApJ, 514, 1

Cen, R., \& Ostriker, J. P. 2005, [arXiv:astro-ph/0601008]

Condon, J. J., Cotton, W. D., Greisen, E. W., et al. 1998, AJ, 115, 1693

Cooray, A. 2002, PhRvD, 65, 103510

Crittenden, R. G., \& Turok, N. 1996, Phys. Rev. Lett., 76, 575

Croft, R. A. C., Weinberg, D. H., Katz, N., \& Hernquist, L. 1998, ApJ, 495, 44

Diaferio, A., Sunyaev, R. A., \& Nusser, A. 2000, ApJ, 533, L71

Diaferio, A., Borgani, S., Moscardini, L., et al. 2005, MNRAS, 356, 1477

Dobbs, M., et al. 2006, New Astron. Rev., 50, 960

Douspis, M., Castro, P. G., Caprini, C., \& Aghanim, N. 2008, A\&A, 485, 395

Dunkley, J., et al. 2008, [arXiv:0803.0586]

Eisenstein, D. J., Annis, J., Gunn, J. E., et al. 2001, AJ, 122, 2267

Fowler, J. W., \& ACT Collaboration 2006, Bull. Am. Astron. Soc., 38, 1227

Fukugita, M., \& Peebles, P. J. E. 2004, ApJ, 616, 643

Génova-Santos, R., Rubiño-Martín, J. A., Rebolo, R., et al. 2005, MNRAS, 363,79

Genova-Santos, R., et al. 2008, [arXiv:0804.0199]

Gorski, K. M. 1994, ApJ, 430, L85

Górski, K. M., Banday, A. J., Bennett, C. L., et al. 1996, ApJ, 464, L11

Górski, K. M., E. Hivon, A. J. Banday, B. D., et al. 2005 ApJ, 622, 759

Hansen, F. K., Branchini, E., Mazzotta, P., Cabella, P., \& Dolag, K. 2005, MNRAS, 361, 753

Hernández-Monteagudo, C., \& Rubiño-Martín, J. A. 2004, MNRAS, 347, 403

Hernández-Monteagudo, C., Genova-Santos, R., \& Atrio-Barandela, F. 2004, ApJ, 613, L89
Hernández-Monteagudo, C., Verde, L., \& Jimenez, R. 2006a, ApJ, 653, 1

Hernández-Monteagudo, C., Verde, L., Jimenez, R., \& Spergel, D. N. 2006b, ApJ, 643, 598

Hernández-Monteagudo, C., Trac, H., Verde, L., \& Jimenez, R. 2006c, ApJ, 652, L1

Hinshaw, G., Spergel, D. N., Verde, L., et al. 2003, ApJSS, 148, 135

Hinshaw, G., et al. 2008, [arXiv:0803.0732]

Hu, W., \& Dodelson, S. 2002, ARA\&A, 40, 171

Itoh, N., \& Nozawa, S. 2004, A\&A, 417, 827

Kamionkowski, M., Kosowsky, A., \& Stebbins, A. 1997, Phys. Rev. D, 55, 7368 Kashlinsky, A., \& Atrio-Barandela, F. 2000, ApJ, 536, L67

Kosowsky, A. 2003, New Astron. Rev., 47, 939

Kull, A., \& Böhringer, H. 1999, A\&A, 341, 23

Larson, G. J., Bunn, E. F., Kasliwal, V., \& McCann, M. 2005, BAAS, 37, 1429

Martinez-Gonzalez, E., Sanz, J. L., \& Silk, J. 1990, ApJ, 355, L5

McDonald, P., Seljak, U., Cen, R., et al. 2005, ApJ, 635, 761

Mortlock, D. J., Challinor, A. D., \& Hobson, M. P. 2002, MNRAS, 330, 405

Nicastro, F., Mathur, S., Elvis, M., et al. 2005a, ApJ, 629, 700

Nicastro, F., Mathur, S., Elvis, M., et al. 2005b, Nature, 433, 495

Peel, A. C. 2006, MNRAS, 365, 1191

Rees, M. J., \& Sciama, D. W. 1968, Nature, 217, 511

Reichardt, C. L., et al. 2008, [arXiv:0801.1491]

Rubiño-Martín, J. A., Atrio-Barandela, F., \& Hernández-Monteagudo, C. 2000, ApJ, 538, 53

Ruhl, J., Ade, P. A. R., Carlstrom, J. E., et al. 2004, SPIE, 5498, 11

Sachs, R. K., \& Wolfe, A. M. 1967, ApJ, 147, 73

Sanz, J., et al. 1999, Evolution of Large Scale Structure: From Recombination to Garching, 53

Seljak, U., \& Zaldarriaga, M. 1996, ApJ, 469, 437

Sheth, R. K., \& Tormen, G. 1999, MNRAS, 308, 119

Smoot, G. F., Bennett, C. L., Kogut, A., et al. 1992, ApJ, 396, L1

Spergel, D. N., Bean, R., Doré, O., et al. 2007, ApJSS, 170, 377

Sunyaev, R. A., \& Zeldovich, Y. B. 1970, Ap\&SS, 7, 3

Sunyaev, R. A., \& Zeldovich, Y. B. 1972, Comm. Astrophys. Space Phys., 4, 173

Sunyaev, R. A., \& Zeldovich, Y. B. 1980, ARA\&A, 18, 537

Tegmark, M. 1997, PhRvD, 55, 5895

Zaldarriaga, M., \& Seljak, U. 1997, Phys. Rev. D, 55, 1830

Zappacosta, L., Mannucci, F., Maiolino, R. 2002, A\&A, 394, 7 